\title{
Neutrophil extracellular traps promote gastric cancer metastasis by inducing epithelial-mesenchymal transition
}

\author{
TONG ZHU ${ }^{1}$, XIAOMING ZOU ${ }^{1}$, CHUNFA YANG $^{2}$, LIANGLIANG LI $^{3}$, BING WANG $^{3}$, RONG $^{1}{ }^{1}$, \\ HONGXUAN LI ${ }^{1}$, ZHANGXUAN XU ${ }^{1}$, DI HUANG ${ }^{3}$ and QINGYUN WU ${ }^{1}$ \\ ${ }^{1}$ The Second Affiliated Hospital of Harbin Medical University, Harbin, Heilongjiang 150086; \\ ${ }^{2}$ Shuangyashan Shuangkuang Hospital, Shuangyashan, Heilongjiang 155100; \\ ${ }^{3}$ Department of Pharmacology, Harbin Medical University, Harbin, Heilongjiang 150086, P.R. China
}

Received March 20, 2020; Accepted June 19, 2020

DOI: $10.3892 /$ ijmm.2021.4960

\begin{abstract}
The risks of tumor recurrence following the successful resection of the primary tumor have been known for decades; however, the precise mechanisms underlying treatment failures remain unknown. The formation of neutrophil extracellular traps (NETs) has increasingly been demonstrated to be associated with thrombi formation in cancer patients, as well as with the development and metastasis of cancer. The present study demonstrated that the level of peripheral blood NETs in patients with gastric cancer (GC) was associated with tumor progression, and patients with stage III/IV disease exhibited significant differences compared with the healthy controls and patients with stage I/II disease, which may be associated with an increased risk of metastasis. In addition, plasma from patients with stage III/IV GC was more prone to stimulate neutrophils to form NETs; thus, it was hypothesized that the formation of NETs may be affected by the tumor microenvironment. A higher deposition of NETs in GC tissues compared with normal resection margins was also identified. In vitro, following treatment with phorbol myristate acetate, which promotes the formation of NETs, or with DNAse-1/GSK-484, which inhibits the formation of NETs, it was found that the tumor migratory ability was altered; however, no significant changes were observed in cell proliferation and cell cycle progression. Epithelial-mesenchymal transition (EMT) is a key event associated with dissemination and metastasis in GC pathogenesis. Finally, the present study demonstrated that NETs promote a more aggressive mesenchymal phenotype and promote the progression of GC in vitro and in vivo. On the whole, to the best of our knowledge, the present study reports a previously unknown role of NETs in the regulation of
\end{abstract}

Correspondence to: Professor Xiaoming Zou, The Second Affiliated Hospital of Harbin Medical University, 148 Bao Jian Road, Harbin, Heilongjiang 150086, P.R. China

E-mail: zou4930@163.com

Key words: gastric cancer, neutrophil extracellular traps, metastasis, epithelial-mesenchymal transition
GC, which is associated with EMT and migration. Therefore, targeting NETs may prove to be therapeutically beneficial.

\section{Introduction}

Gastric cancer (GC) is the fifth most frequently diagnosed malignancy and the third leading cause of cancer-associated mortality worldwide (1). According to cancer statistics in China in 2015, GC is the second and the third most commonly diagnosed type of cancer among males and females, respectively, in the Chinese population (2). Despite improvements in the clinical treatment of GC in recent years, the survival rate of patients with GC remains $<30 \%$ (2). As researchers spare no efforts to study cancer progression, views on tumor development have markedly changed, and the malignant cells themselves been shown to make up a complex area, termed the tumor microenvironment (TME) (3). Recent evidence has indicated that the crosstalk between tumor cells and the TME is essential for tumor progression, invasion and metastasis (4).

Inflammation is regarded as one of the hallmarks of cancer; inflammatory cells and associated factors play an essential role in almost every stage of tumor progression, including metastasis (5). Neutrophils, the most abundant type of white blood cells in the peripheral bloodstream, are important components of tumor-associated infiltrating inflammatory and immune cells in GC (6). Neutrophils used to be considered inert bystander cells in cancer progression and development, however this view has changed $(7,8)$. Advances in neutrophil biology have revealed that neutrophils can release their decondensed chromatin and form large extracellular DNA networks, termed neutrophil extracellular traps (NETs). Intensive investigations on NETs have demonstrated a potential involvement of NETs in neoplastic disease. The pro-tumor and antitumor effects of NETs have been described (9). It has been reported that tumor cells and cancer cell-primed platelets can promote the release of NETs by host neutrophils $(10,11)$. In patients with Ewing's sarcoma, the presence of tumor-associated neutrophils and NETs is only detected in patients with metastasis, indicating that NETs may promote tumor progression (12). In a study comparing human triple-negative breast cancer with luminal and HER-2 $2^{+}$breast cancer, it was identified that the presence of NETs was associated with the metastatic burden, 
and the triple-negative breast cancer cases exhibited the highest number of NETs (13). Furthermore, it has been observed that circulating NETs, produced upon stimulation in infectious and non-infectious diseases, capture circulating tumor cells, which then promotes tumor progression and metastasis in vitro and in vivo (14). The degradation or inhibition of NETs by DNAse-1 or neutrophil elastase (NE) inhibitor has been demonstrated to prevent metastasis in a murine cecal ligation and puncture model (15). Citrullinated histone H3 (cit-h3; a NETs activation marker) is detectable in the plasma of patients with advanced cancer, and can serve as a distinct prognostic marker that is associated with a poor clinical outcome in numerous types of tumor (16). After deposition of NETs was found to be significant in malignant tumors, it was also identified that NETs increase the risk of thrombosis in patients with cancer, which may be associated with a poor prognosis $(17,18)$. Conversely, the antitumor effects of NETs have also been reported in colon cancer and melanoma. Following the stimulation of tumor cells with NETs, the ability to metastasize and proliferate is decreased $(19,20)$. In addition, NETs may act as a double-edged sword in cancer biology and the effects of NETs on tumor cells may vary in different types of tumors. To the best of our knowledge, there is no clear conclusion on the interaction between NETs and tumors or whether NETs function as a pro-tumor or antitumor factor. The present study aimed to provide evidence to uncover the role of NETs in GC progression.

Metastatic progression is regulated by alterations in the TME, such as inflammation, angiogenesis, and cancerized stroma and intravasation, which is termed epithelial-mesenchymal transition (EMT) (21). EMT in tumor progression allows a polarized epithelial cell to acquire a mesenchymal cell phenotype, and it facilitates the intravasation of tumor cells into blood or lymph vessels and the subsequent formation of distant metastasis (22). Neutrophils have been observed to promote the EMT process (23); however, the underlying mechanisms remain unclear. Related factors released by neutrophils, such as interleukin (IL)-17, can promote the EMT process of tumor cells via the JAK2/STAT3 pathway (24). NETs have been reported to be able to promote tumor cell adhesion and thus promote tumor cell progression and metastasis (25). In the present study, a large amount of NETs formation was found in the plasma of patients with advanced GC and in GC tumor tissues; however, the interaction between tumor and NETs needs to be further investigated. The aim of the present study was to reveal the role of NETs in the progression and metastasis of GC.

\section{Materials and methods}

Patients. Patients with gastric cancer and healthy control subjects were recruited at the Department of Gastrointestinal Surgery in the Second Affiliated Hospital of Harbin Medical University of China, between July, 2017 to December, 2019. Patients were diagnosed with GC by a histological diagnosis which involved post-operative pathology and tumor-node-metastasis (TNM) staging was assessed according to the 8th American Joint Committee on Cancer (AJCC) Staging Classification Guidelines (26). The exclusion criteria were as follows: An age $<18$ years, pregnancy, cardiovascular disease, diabetes, active or chronic infection, liver or renal dysfunction, other coexisting cancers, platelets and/or blood coagulation disorders, and the administration of anticoagulant and/or anti-platelet treatment. The main characteristics of the patients and healthy controls are presented in Table I. The present study was approved by the Research Ethics Committee of Second Affiliated Hospital of Harbin Medical University, all subjects manually signed the informed consent.

Cells and cell culture. The human GC cell line, AGS, was obtained from the Cell Bank of Chinese Academy of Science. Cells were cultured in RPMI-1640 medium (\#31870082, Gibco; Thermo Fisher Scientific, Inc.) containing $10 \%$ fetal bovine serum (FBS, \#04-001-1ACS, BI) supplemented with penicillin $(100 \mathrm{U} / \mathrm{ml})$ and streptomycin (\#ST488, Beyotime Institute of Biotechnology, Inc.; $100 \mathrm{U} / \mathrm{ml}$ ) at $37^{\circ} \mathrm{C}$ with $5 \% \mathrm{CO}_{2}$ in a humidified atmosphere.

Isolation of neutrophils. Fresh peripheral blood was obtained from patients with GC and healthy controls using the commercial EDTA-k2 anticoagulant tubes routinely used at the Second Affiliated Hospital of Harbin Medical University. Blood samples were obtained within $2 \mathrm{~h}$ prior to isolation. Human neutrophils were isolated using polymorphprep ${ }^{\mathrm{TM}}$ (27). Briefly, $5 \mathrm{ml}$ of fresh whole blood were layered on top of $5 \mathrm{ml}$ of PolymorphPrep (\#1114683, Axis-Shield) in a $15 \mathrm{ml}$ tube and centrifuged at $500 \times \mathrm{g}$ for $30 \mathrm{~min}$ at room temperature. The lower leukocyte band containing neutrophils was collected, washed and resuspended into $5 \mathrm{ml}$ of ACK lysis buffer (\#C3702, Beyotime Institute of Biotechnology, Inc.), washed twice in HBSS without $\mathrm{Ca}^{2+} / \mathrm{Mg}^{2+}$, and finally resuspended in serum-free RPMI-1640 (\#31870082, Gibco; Thermo Fisher Scientific, Inc.).

Stimulation and inhibition of NET formation. Purified neutrophils $(500,000)$ isolated from GC patients or healthy controls were subsequently incubated at $37^{\circ} \mathrm{C}$ in $5 \% \mathrm{CO}_{2}$ then activated overnight with $25 \mathrm{nM}$ PMA (\#P1585, phorbol 12-myristate 13-acetate, Sigma-Aldrich; Merck KGaA). In the inhibition assay, PMA and the inhibitors were used in combination. NETs inhibitors were added $30 \mathrm{~min}$ prior to neutrophil activation to inhibit NETs formation. The PAD4 inhibitor, GSK484 (\#17488, Cayman Chemical Company) was used at $10 \mu \mathrm{M}$ to inhibit NETs formation, and 1.5 units/ml of DNase I (\#04536282001, Sigma-Aldrich; Merck KGaA) was used to digest NETs scaffolds. The supernatant containing NETs was collected and centrifugation at $250 \mathrm{x} \mathrm{g}$ for $10 \mathrm{~min}$ at room temperature. Isolated NETs were stored at $-80^{\circ} \mathrm{C}$ until further use as previously reported (28). To investigate the effect of the TME on NETs formation, neutrophils were treated using $20 \%$ plasma from the same patients with GC $(n=20)$ or the controls $(n=10)$ as above, following a previously described protocol (29).

Immunofluorescence in neutrophils. To further assess NETs formation in vitro, neutrophils were seeded on poly-L-lysine-coated coverslips (\#354085, Corning, Inc.) and stimulated with PMA for $3 \mathrm{~h}$ and then fixed with $4 \%$ paraformaldehyde (PFA, \#BL539A, Biosharp) for $20 \mathrm{~min}$ at room temperature, rinsed twice in PBS, incubated in $50 \mathrm{mM}$ of $\mathrm{NH}_{4} \mathrm{Cl}$ for $10 \mathrm{~min}$ at room temperature and permeabilized 


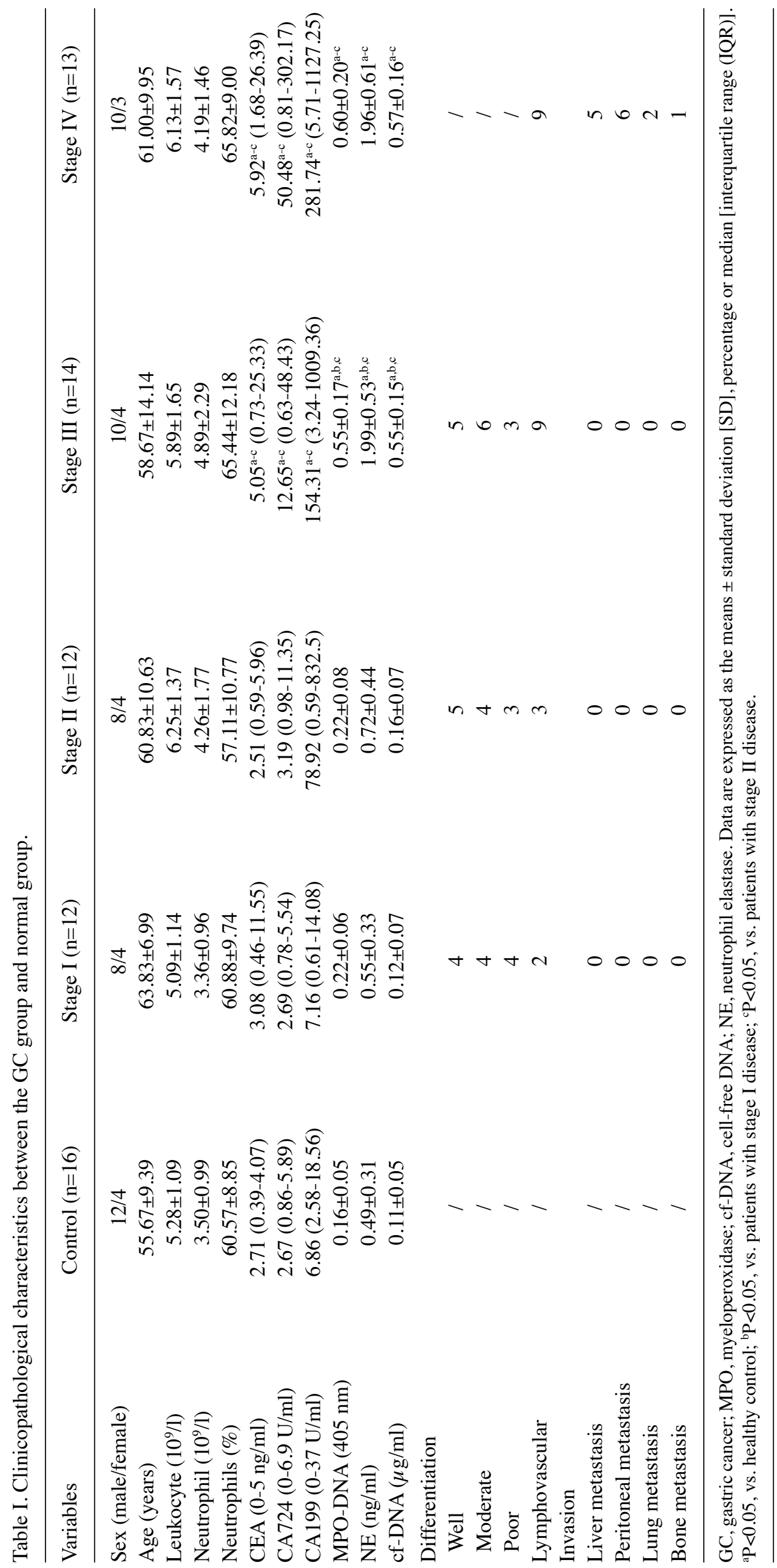


with $0.5 \%$ Triton X-100 (\#BB151-500, Thermo Fisher Scientific, Inc.) for $1 \mathrm{~min}$ at room temperature. The cells were then blocked in PBS containing $1 \%$ bovine serum albumin (BSA, \#A3294, Sigma-Aldrich; Merck KGaA) for $30 \mathrm{~min}$ at $37^{\circ} \mathrm{C}$ and incubated with neutrophil elastase (NE) antibody (\#sc-55549, Santa Cruz Biotechnology, Inc.) at 1:400 dilution in blocking buffer for $1 \mathrm{~h}$ at room temperature. Following washes 3 times in PBS, the cells were incubated in the presence of fluorochrome-conjugated secondary antibodies (\#A11001, Alexa Fluor 488, Invitrogen; Thermo Fisher Scientific, Inc.) at 1:200 dilution at room temperature for $1 \mathrm{~h}$, rinsed twice in PBS, stained with Hoechst 33342 (\#C1022, Beyotime Institute of Biotechnology) at 1:5 dilution at room temperature for 5 min, rinsed in PBS, and the coverslips were mounted onto glass slides using a fluorescence microscope (DM400B, Leica Microsystems $\mathrm{GmbH}$ ). The percentage of NETs was evaluated by counting the number of NET-releasing neutrophils out of the total number of neutrophils.

Measurement of the formation of NETs in patients with GC. Circulating cell-free DNA (cfDNA), NE and MPO-DNA complex, were all measured as a presence for NETs in the subjects' plasma $(30,31)$. The Quant-iT PicoGreen dsDNA Assay kit (\#P11496, Invitrogen; Thermo Fisher Scientific, Inc.) was used to quantify the GC patient cfDNA levels in plasma as per the provided directions. Human plasma NE was quantified using selective ELISA (\#MM-1433H1, Jiangsu Meimian Industrial Co., Ltd.) based on provided instruction. To quantify the MPO-DNA complex in the plasma, a capture ELISA was used as previously described (32). In brief, $5 \mu \mathrm{g} / \mathrm{ml}$ anti-MPO antibody (\#sc-52707, Santa Cruz Biotechnology, Inc.) was coated onto 96-well plates (dilution 1:500 in 50 $\mu \mathrm{l}$ ) at $4^{\circ} \mathrm{C}$ overnight. After washing with PBS 3 times $(300 \mu \mathrm{l}$ each), $20 \mu \mathrm{l}$ of samples were added to the wells with $80 \mu \mathrm{l}$ incubation buffer containing a peroxidase-labeled anti DNA mAb (cat. no. 11774425001, Cell Death ELISA ${ }^{\text {plus }}$, Roche Diagnostics; dilution, 1:25). The plate was incubated for $2 \mathrm{~h}$, and shaking at $300 \mathrm{rpm}$ at room temperature. Following 3 washes with PBS (300 $\mu \mathrm{l}$ each), $100 \mu l$ peroxidase substrate (ABST) of the kit was added. The absorbance at $405 \mathrm{~nm}$ wavelength was measured following $20 \mathrm{~min}$ of incubation at room temperature in the dark.

Cell cycle analysis. Following treatment with NETs or NETs inhibitors for $24 \mathrm{~h}$, the AGS cells were washed with cold PBS and then fixed overnight with $70 \%$ cold ethanol at $-20^{\circ} \mathrm{C}$. The cells were then washed again with cold PBS and incubated in the dark with propidium iodide (PI, \#ST511, Beyotime Institute of Biotechnology) staining solution containing RNase A for $30 \mathrm{~min}$ at room temperature. The cell cycle was measured using a FACSCanto II flow cytometer (BD Biosciences). The proportion of cells in the growth 0 /growth $1(\mathrm{G} 0 / \mathrm{G} 1)$, synthesis (S) and growth $2 /$ mitosis $(\mathrm{G} 2 / \mathrm{M})$ phase were calculated using FlowJo software programs (version 10), and are represented as DNA histograms.

Migration assay. For the migration assay, $2 \times 10^{5}$ cancer cells were loaded into the upper chamber of a cell culture insert with $8 \mu \mathrm{M}$ pore size (\#3422, Corning Inc.) with NETs culture medium (CM) with or without NETs inhibitors and the lower chamber was supplemented with cell culture medium containing $10 \% \mathrm{FBS}$. Following $24 \mathrm{~h}$ of incubation at $37^{\circ} \mathrm{C}$, the migrated cells were fixed and stained with crystal violet staining solution (\#G1063, Beijing Solarbio Science \& Technology Co., Ltd.) at room temperature and were counted under a microscope (Olympus Corp.).

MTT assay. The AGS cells were plated in 96-well plates at a density of $3 \times 10^{3}$ per well. After the cells had reached approximately $80 \%$ confluency, they were starved overnight. The cells were then exposed to NETs CM with or without inhibitors for 24 and $48 \mathrm{~h}$, respectively. Following incubation for 24 or $48 \mathrm{~h}$ at $37^{\circ} \mathrm{C}$, MTT (\#M2128, Sigma-Aldrich; Merck KGaA; $20 \mu \mathrm{l} /$ well) solution was added, and the cells were incubated for $6 \mathrm{~h}$ at $37^{\circ} \mathrm{C}$. The medium in the plate was then discarded, and $150 \mu \mathrm{l}$ dimethylsulfoxide (DMSO) were added to each well. The absorbance was then measured at $570 \mathrm{~nm}$ using the PowerWave HT microplate spectrophotometer (BioTek Instruments, Inc.).

Post-surgical residual tumor xenograft models. Male athymic BALB/c nude mice ( $\mathrm{n}=20$, 5-6 weeks old, weighing 20-25 g) were purchased from Beijing Vital River Laboratory Animal Technology Co. Ltd. [Certificate no. SCXK (Jing) 2016-0011 (no. 11400700161156)]. All animal experiments were performed in accordance with the NIH guidelines (Guide for the Care and Use of Laboratory Animals) and approved by the Ethics Committee of the Second Affiliated Hospital of Harbin Medical University. The Ethics Committee of the Second Affiliated Hospital of Harbin Medical University approved the present study (permit no. KY2016-032). Mice were kept in a controlled SPF environment at $23 \pm 2{ }^{\circ} \mathrm{C}, 40-70 \%$ humidity under a $12 \mathrm{~h}$ dark/light cycle with free access to irradiated food and sterile water. They were housed in individually ventilated cages: 5 per cage, with 4-6 mm corncob bedding following disinfection with ${ }^{60} \mathrm{Co}$ radiation. A murine model was established with a post-surgical residual tumor to mimic the recurrence of a solid tumor. The AGS tumor cells $\left(>5 \times 10^{7} / \mathrm{ml}\right)$ were harvested and washed with saline. A cell suspension of $1 \times 10^{6}$ in $0.2 \mathrm{ml}$ was injected subcutaneously into the right axillary of the mice to establish the traditional tumor xenograft nude mouse model $(n=5)$. When the tumors reached an average volume of $1,000-1,500 \mathrm{~mm}^{3}$, the tumors were removed from the mice aseptically and diced into small cubes $(2.0 \times 2.0 \times 2.0 \mathrm{~mm})$. These tumor chunks were then injected subcutaneously into the right axillary of the new mice $(n=15)$ to establish a murine model with a post-surgical residual tumor. When the tumors had reached an average volume of $300-350 \mathrm{~mm}^{3}$, the mice were anesthetized by intraperitoneal injection of $1.2 \%$ avertin (2, 2, 2-tribromoethanol) solution $(260 \mathrm{mg} / \mathrm{kg})$. A small incision was made to remove tumor tissues around the tumor edge aseptically, leaving a residual tumor volume of $60-100 \mathrm{~mm}^{3}$. After the surgery, the mice were divided into 3 groups $(n=5)$ based on the tumor volume. Body weight was measured twice a week. The mice in the NETs inhibition treatment groups were administered daily with DNAse-1 or GSK484 by an intraperitoneal injection at a dose of 15,000 units $/ \mathrm{kg}$ DNAse- 1 or $20 \mathrm{mg} / \mathrm{kg}$ GSK484 for 14 days. The control mice were treated with $0.1 \%$ DMSO in $100 \mu 1$ saline. Tumor diameters was measured in two dimensions 
A

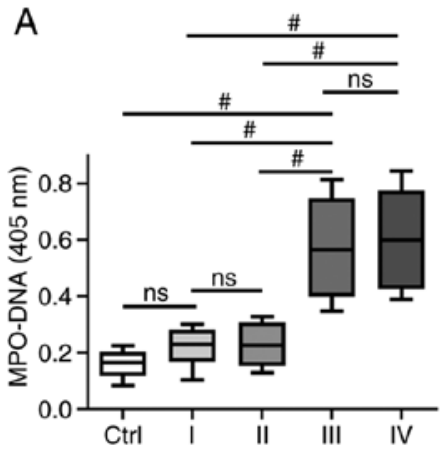

B

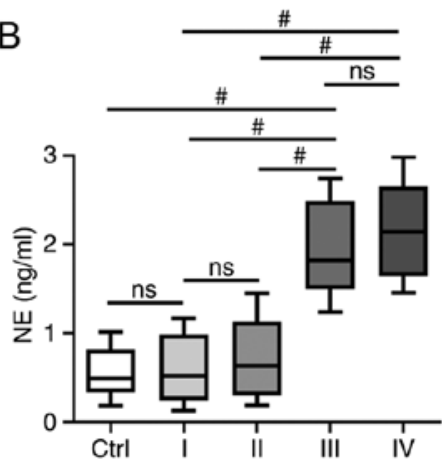

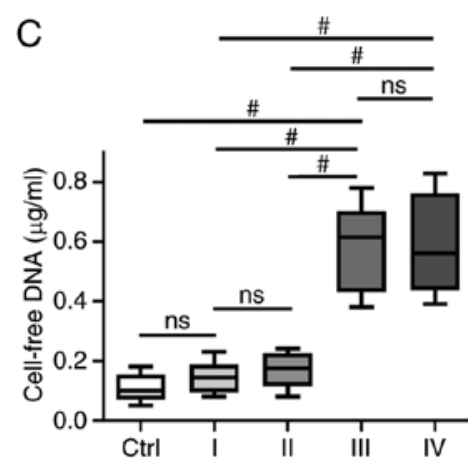

Figure 1. Circulating NET markers in healthy controls and patients with GC. (A-C) MPO-DNA complexes, NE and cell-free DNA of healthy subjects and patients with GC are shown, respectively. Data are presented as the median (horizontal bar), 25th and 75th percentile (boxes), and 10th and 90th percentile (error bar). ns, not significant, ${ }^{\#} \mathrm{P}<0.001$. NET, neutrophil extracellular trap; MPO, myeloperoxidase; NE, neutrophil elastase.

every 2-3 days. Tumor volume was calculated according to the formula: Volume $\left(\mathrm{mm}^{3}\right)=\left(\right.$ width $^{2} \mathrm{x}$ length $) / 2$. The mice were sacrificed at 14 days after the injection of DNAse-1 or GSK484. At the end of the experiments, the mice were euthanized by cervical dislocation. Tumors were excised, weighed, and fixed in $4 \%$ paraformaldehyde at room temperature for the paraffin sections or stored at $-80^{\circ} \mathrm{C}$.

Western blot analysis. Whole cell lysates were extracted using RIPA lysis buffer (\#P0013C, Beyotime Institute of Biotechnology, Inc.) with protease inhibitor (Roche Diagnostics). The quantities of protein were determined using a bicinchoninic acid kit (\#P0012, Beyotime Institute of Biotechnology). Protein samples of $100 \mu \mathrm{g}$ per lane were loaded onto $10 \%$ SDS-PAGE gels and transferred onto nitrocellulose membranes (PALL) for $90 \mathrm{~min}$. For immunodetection, the membranes were incubated with the following primary antibodies at $4^{\circ} \mathrm{C}$ overnight: E-cadherin (\#ab76055, Abcam) at a 1:1,000 dilution, vimentin (\#HPA001762, Sigma-Aldrich; Merck KGaA) at a 1:1,000 dilution, and cit-h3 (\#ab5103, Abcam), GAPDH (\#60004-1-Ig, Proteintech) at a 1:1,000 dilution. The fluorescence-conjugated secondary IRDye 800 mouse (\#962-32210) and rabbit antibodies (\#962-32211) were purchased from LI-COR. Following incubation with secondary antibody at 1:7,500 dilution with gentle shaking at room temperature for $60 \mathrm{~min}$, the membranes were scanned by Odyssey Infrared Imaging System (LI-COR Biosciences). Protein expression was quantified using Image-Pro ${ }^{\circledR}$ Plus software (version 6.0; Media cybernetics, Inc.). All experiments were performed at least 5 times.

Immunohistochemistry. For immunohistochemistry, heat-induced epitope retrieval was performed by Citrate Antigen Retrieval solution (\#C1031, Beijing Solarbio Science \& Technology Co., Ltd.) for $40 \mathrm{~min}$ for vimentin, or by EDTA Antigen Retrieval solution (\#ZLI-9079, ZSGB-Bio) for E-cadherin. The tissue preparations were incubated with the primary antibodies for E-cadherin (\#20874-1-AP, ProteinTech Group, Inc.,) at a 1:500 dilution, vimentin (\#HPA001762, Sigma-Aldrich; Merck KGaA) at a 1:250 dilution at $4^{\circ} \mathrm{C}$ overnight, followed by incubation with the secondary antibody EnVision $^{\mathrm{TM}}+$ /HRP rabbit polymer (\#P0448, Dako; Agilent Technologies, Inc.) at a 1:200 dilution at room temperature for
$30 \mathrm{~min}$. Secondary antibody detection was performed by using the SIGMAFAST ${ }^{\mathrm{TM}}$ 3,3'-diaminobenzidine tablets (DAB Peroxidase Substrate Tablet Set, \#D4168, Sigma-Aldrich; Merck KGaA). The slides were counterstained with hematoxylin for 2 min following color separation by $1 \%$ acetic acid at room temperature for $30 \mathrm{sec}$. The Samples were visualized under a light microscope (Nikon, model Eclipse E400) and images were captured using a Nikon Digital Camera (ACT-1 Nikon software).

Immunofluorescence in tissues. To detect NETs by immunofluorescence, sections were stained as previously described (33). Non-specific binding sites were blocked with $2 \%$ goat serum in $2 \%$ BSA-PBS. In human tissue staining, NETs were counterstained with a rabbit anti-citrullinated histone $\mathrm{H} 3$ polyclonal antibody (\#ab5103, Abcam) at 1:200 dilution, a mouse anti-NE monoclonal antibody (\#sc-55548, Santa Cruz Biotechnology, Inc.) at 1:200 dilution as the primary antibodies at $4^{\circ} \mathrm{C}$ overnight. The samples were incubated with goat anti-mouse IgG $(\mathrm{H}+\mathrm{L})$ Highly Cross-Adsorbed Secondary Antibody, Alexa Fluor 488 (\#A-11032, Invitrogen ${ }^{\mathrm{TM}}$, Thermo Fisher Scientific, Inc.), or goat anti-rabbit IgG (H+L) Highly Cross-Adsorbed Secondary Antibody, Alexa Fluor 594 (\#A32740, Invitrogen ${ }^{\mathrm{TM}}$, Thermo Fisher Scientific, Inc.) at a 1:200 dilution at room temperature for $1 \mathrm{~h}$. Nuclei were stained with 4',6-diamidino-2-phenylindole (\#C1002, Beyotime Institute of Biotechnology) at 1:5 dilution at room temperature for $5 \mathrm{~min}$. The NETs staining methods for the mouse tumor tissues were consistent with those in human tissues, except that antibody species were different. For briefly, NETs were counterstained with a rabbit anti-citrullinated histone H3 antibody (\#ab5103, Abcam) at 1:200 dilution, a rabbit anti-NE antibody (\#AF-0010, Affinity Biosciences) at a 1:200 dilution as the primary antibodies at $4^{\circ} \mathrm{C}$ overnight. $\mathrm{F}\left(\mathrm{ab}^{\prime}\right) 2$-goat anti-rabbit IgG $(\mathrm{H}+\mathrm{L})$ Alexa Fluor 488 antibody (\#A11070, Invitrogen $^{\mathrm{TM}}$, Thermo Fisher Scientific, Inc.) and F(ab')2-goat anti-rabbit IgG (H+L) Alexa Fluor 594 antibody (\#A-11072, Invitrogen $^{\mathrm{TM}}$, Thermo Fisher Scientific, Inc.) were used at a 1:500 dilution at room temperature for $45 \mathrm{~min}$. Mouse sections staining followed the method as previously reported (34). NETs were defined as co-localized DNA, NE and citrullinated histone $\mathrm{H} 3$ (cit-h3) after acquiring images with a laser scanning confocal microscope (LSCM; Olympus Corp.). 


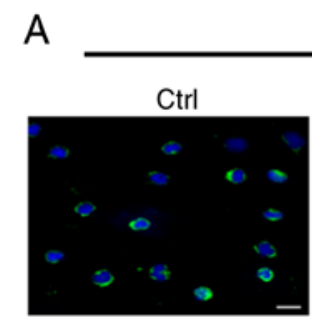

Plasma+neutrophils

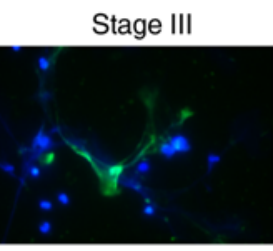

\section{Stage I}

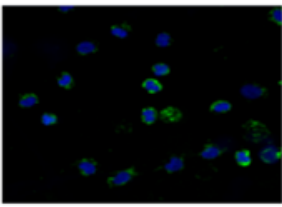

Stage IV

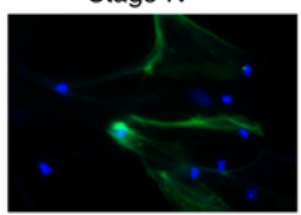

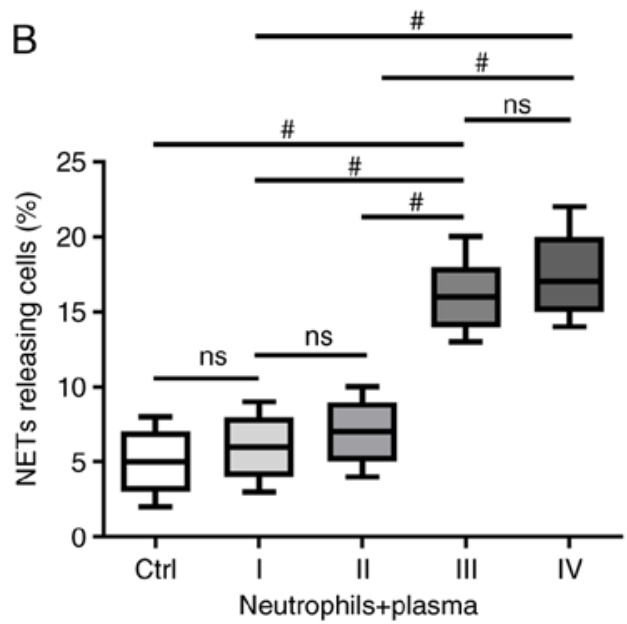

Figure 2. Plasma from patients with advanced GC is more prone to stimulate NET formation. (A) Representative immunofluorescence images showing NETs derived from neutrophils that were stimulated by plasma from healthy controls and GC patients with different TNM stages. NETs were characterized by DNA (Hoechst 33342, blue) and neutrophil elastase (NE, green). Scale bar, $20 \mu \mathrm{m}$; magnification, $\mathrm{x} 400$. (B) Percentage of cells showing release of NETosis neutrophils conditioned using plasma from each group. These results were representative of 10 experiments. Data are the median and IQR and error bars represent the 10th and 90th percentiles. ns, no significant, ${ }^{\prime \prime} \mathrm{P}<0.001$. NET, neutrophil extracellular trap; GC, gastric cancer.
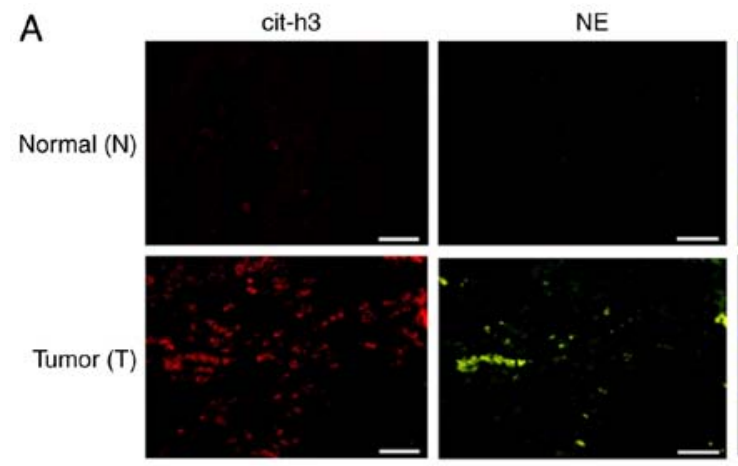

B

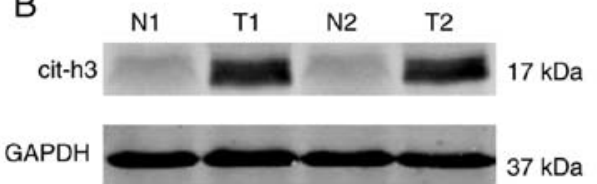

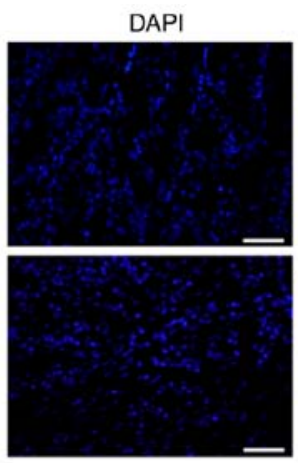
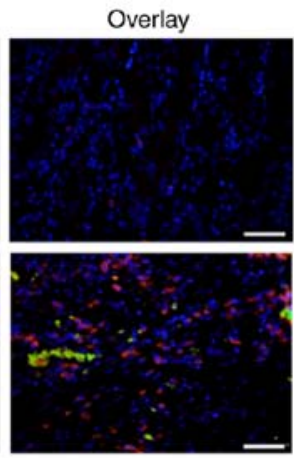

Cit-h3

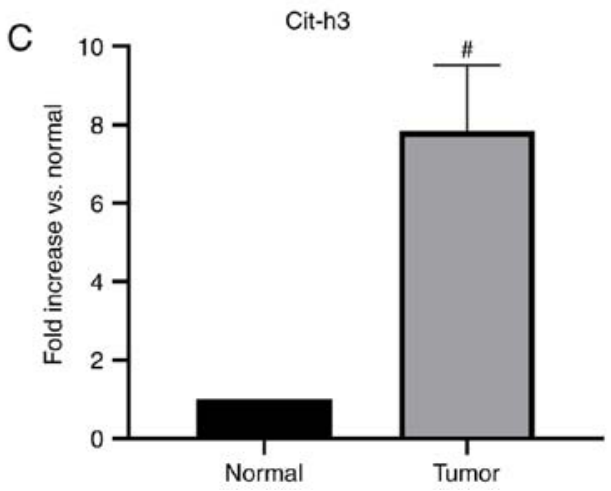

Figure 3. Abundant NET deposition in human GC tissues. (A) NETs were visualized in GC tissues as extracellular structures decorated with neutrophil elastase (green) and citrullinated H3 (red) co-localizing with DAPI/DNA (blue), but the lack of NETs in normal resection edge. Scale bar, $50 \mu$ m; magnification, $\mathrm{x} 400$. (B) Western blot analysis results revealed that the level of cit-H3 was increased in the gastric cancer tissues but nearly absence in normal gastric tissues. The blots are representatives of 3 experiments from 10 pairs of patients with similar results. (C) The values of protein band densities were normalized to cit-h3 protein level of normal group. Data are shown as the means \pm SEM, ${ }^{*} \mathrm{P}<0.001$ vs. normal group. NET, neutrophil extracellular trap; GC, gastric cancer; cit-h3, citrullinated histone $\mathrm{H} 3$.

Statistical analysis. Statistical analysis was performed using GraphPad Software 5.0 (GraphPad Software, Inc.). Comparisons between 2 groups were conducted using a Student's t-test. Differences between multiple groups were determined by one-way ANOVA with Tukey's post hoc test. A probability value of $\mathrm{P}<0.05$ was considered to indicate a statistically significant difference.

\section{Results}

Comparison of the clinicopathological characteristics of patients with different stages of GC. A total of 51 patients with GC, including 12 cases at stage I, 12 cases at stage II, 14 cases at stage III and 13 cases at stage IV, along with 16 healthy controls were recruited in this prospective study. The patient 

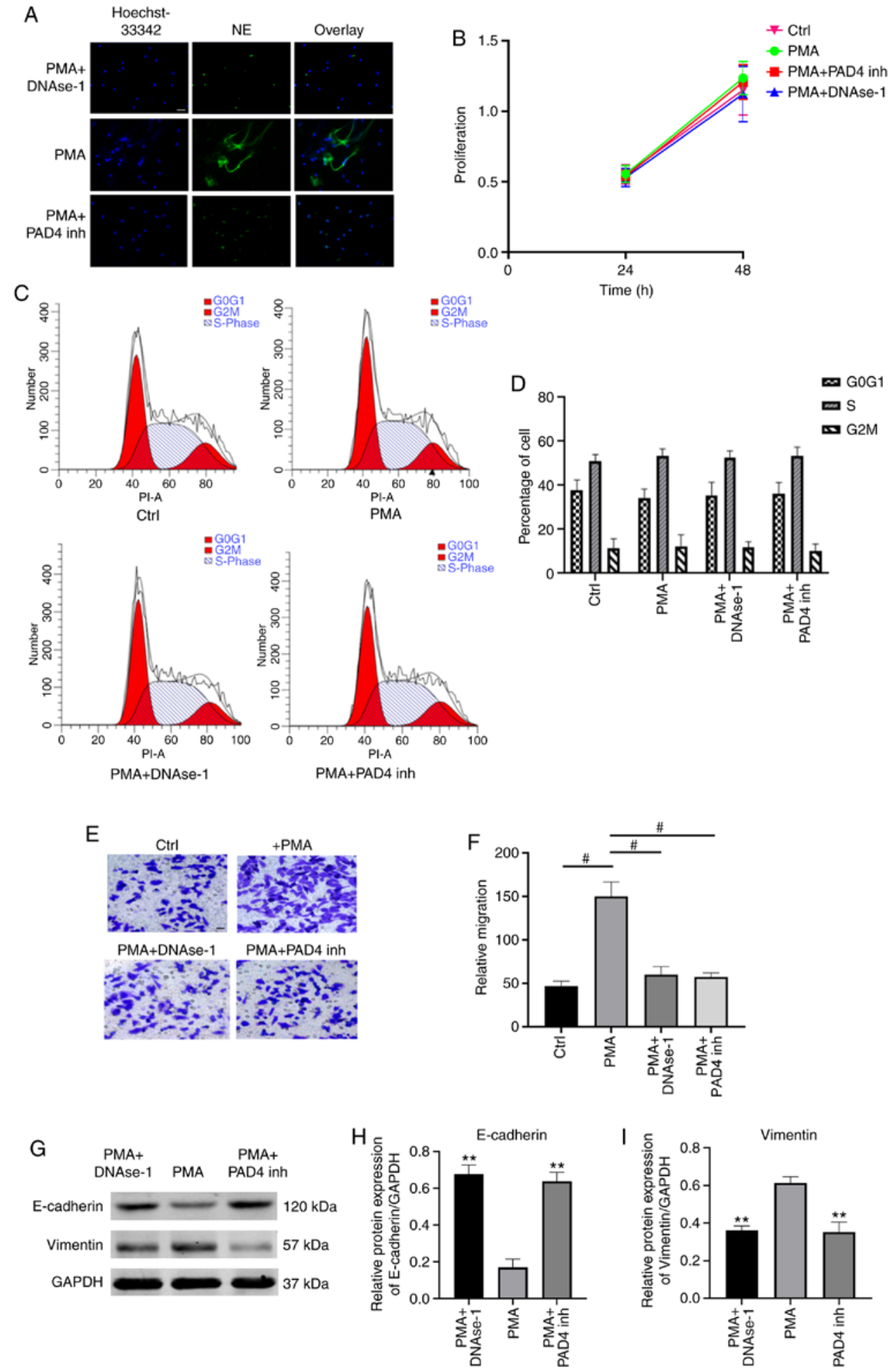

Figure 4. NETs promote tumor cell migration without altering cell cycle and proliferation. (A) Representative immunofluorescence microphotographs showing NETs (Hoechst-44432, blue; NE, green). Scale bar, $50 \mu \mathrm{m}$; magnification, x200. (B) MTT assay indicated that there was no significant change in the proliferation rate of AGS cells for $48 \mathrm{~h}$ in each group. Data are presented as the means \pm SEM from $n=3$ separate experiments. (C) The effects of NETs on cell cycle was assessed by flow cytometry, AGS cells were stimulated by NETs or inhibiting NET CM for $24 \mathrm{~h}$. (D) The percentage of cell population at each phase in different group. (E) The effects of NETs on the migration ability of GC cells was assessed by Transwell assay; scale bar, $20 \mu$ m; magnification, x400. (F) Statistical graphs are presented as the means $\pm \mathrm{SEM},{ }^{\#} \mathrm{P}<0.001 ; \mathrm{n}=5$. (G) The effects of NETs on the EMT markers in AGS cells and GAPDH were examined by western blot analysis. (H and I) Statistical analysis of the expression of E-cadherin and vimentin was showed. Data were normalized to the GAPDH protein level and are expressed as the mean $\pm \mathrm{SEM},{ }^{* *} \mathrm{P}<0.01$ vs. PMA group; $\mathrm{n}=3$. NET, neutrophil extracellular trap; GC, gastric cancer.

clinical information is presented in Table I and no significant differences were observed in age, sex, and leukocyte and neutrophil counts among the different groups $(\mathrm{P}>0.05)$. Peritoneal metastasis was present in $46.1 \%$ of patients with stage IV GC, liver metastasis was found in $38.5 \%$ ( 2 cases of liver metastasis were accompanied by peritoneal metastasis), and metastasis in 

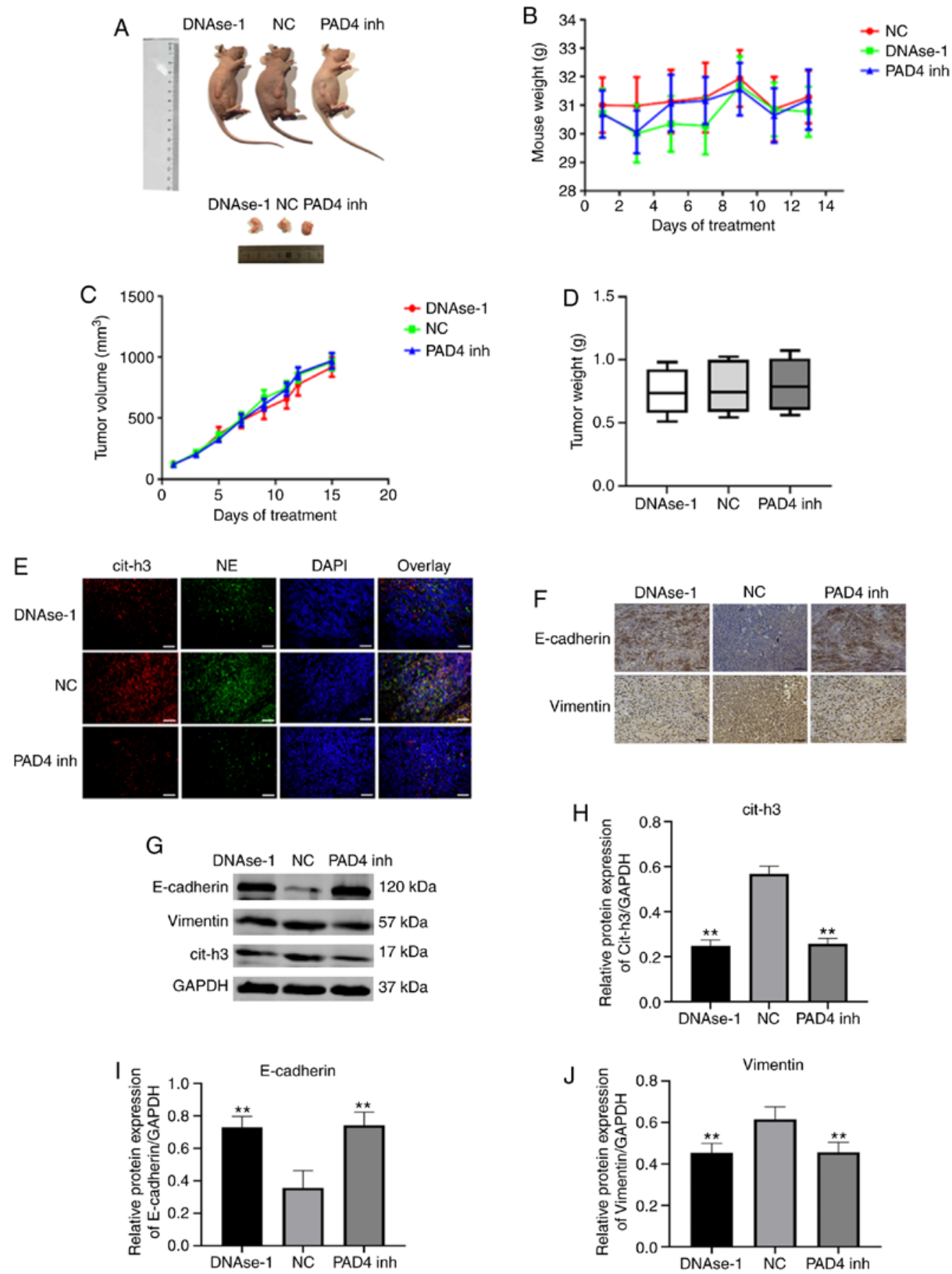

Figure 5. Effects of NET inhibitors on solid tumor growth in nude mice. (A) Images of AGS residual tumor xenografts treated with Dnase I or GSK-484; scale bar, $1 \mathrm{~cm}$. (B-D) The graphs of mouse weight, tumor growth curve, average tumor weight. AGS cells induced NETs deposition in the tumors. (E) Images showing representative immunostaining for neutrophil elastase (green), citrullinated histone H3 (red), and DAPI (blue) in the tumors of mice treated as indicated; scale bar, $50 \mu \mathrm{m}$; magnification, $\mathrm{x} 400$. (F) Representative IHC images of murine tumor sections stained for the EMT-related markers; $\mathrm{n}=5$. scale bar, $50 \mu \mathrm{m}$; magnification, $\mathrm{x} 400$. (G) The effects of NETs inhibitors on EMT process in murine tumor tissues, protein levels of E-cadherin, vimentin and citrullinated histone $\mathrm{H} 3$ were detected by western blot analysis. (H-J) Statistical analysis of the expression of E-cadherin, vimentin and citrullinated histone H3. Data were normalized to the GAPDH protein level and expressed as the means \pm SEM, ${ }^{* *} \mathrm{P}<0.01$ vs. NC group, $\mathrm{n}=5$. NET, neutrophil extracellular trap; GC, gastric cancer; cit-h3, citrullinated histone $\mathrm{H} 3$.

other organs was present in $23 \%$ of cases. In the present study, the overall incidence of lymphovascular invasion (LVI) in all enrolled patients was $45 \%$, with a positive rate of $64.3 \%$ in patients with stage III and $69.2 \%$ in patients with stage IV GC. stage in GC. NETs are extracellular web-like structures of
DNA decorated with various antimicrobial proteins, such as neutrophil-derived NE and myeloperoxidase (MPO). To identify NETs formation in plasma, the levels of different NETs markers, such as circulating MPO-DNA complexes, cf-DNA and NE were assessed. It was identified that the levels of MPO-DNA complexes, cf-DNA and NE were higher in patients with stage III/IV GC $(\mathrm{P}<0.001)$ compared with patients with stage I/II 


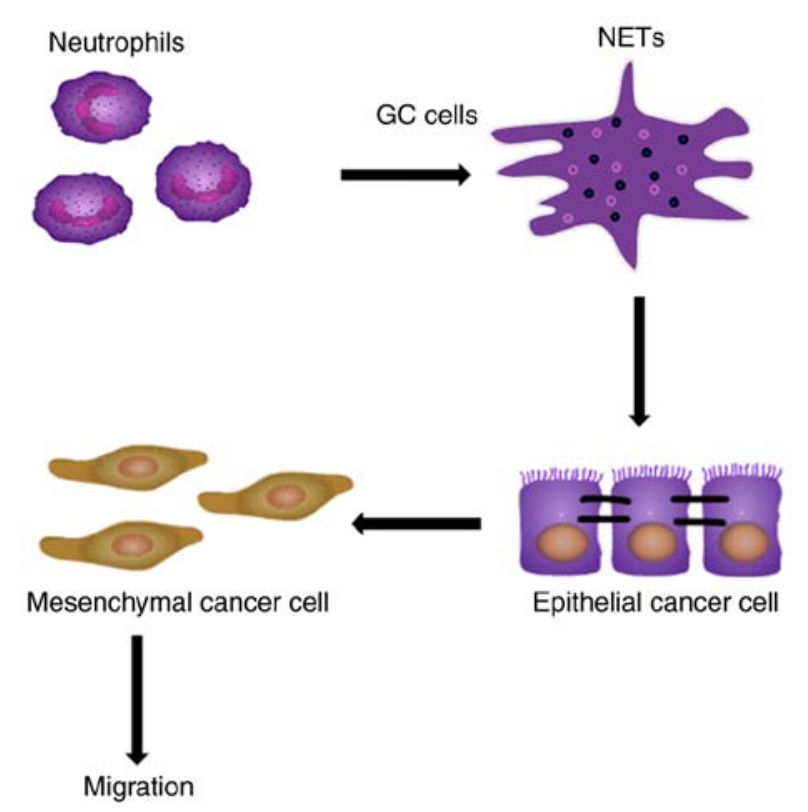

Figure 6. Schematic illustration of the role of NETs in promoting EMT in tumor microenvironment. GC cells could promote neutrophils to develop NETosis, which leads to large amounts of NETs formation. Tumor metastasis may be initiated when abundant NETs formation, activating the EMT process to promote epithelial cells lose their cell-cell contacts and transform to mesenchymal cells expressing mesenchymal markers gaining high migration properties, which contribute to their aggressive tumor phenotype. GC cells can increase their migration ability through EMT after stimulated by NETs, and NETs may also increase the invasion, adhesion and metastasis of GC cells, thereby permitting them to distant metastasis including the liver and peritoneal. NETs, neutrophil extracellular traps; GC, gastric cancer.

GC or the healthy controls; however, no significant difference was observed between patients with stage I/II GC and the healthy controls. In addition, no significant difference was identified in NETs markers, such as MPO-DNA complex, cf-DNA and NE in patients GC with stage III and IV disease (Fig. 1).

TME in patients with advanced GC primes neutrophils to form NETs. To investigate whether the TME of GC induces neutrophils to form NETs, the extracellular co-localization of DNA and granule proteins were observed by staining with Hoechst 33342 and NE. NETs exhibited an extracellular fiber-like structure protruding from neutrophils stimulated with plasma from patients with stage III and IV GC (Fig. 2A). However, the effect was not significant when compared with neutrophils stimulated with plasma from healthy individuals or patients with stage I/II GC (Fig. 2A). The percentage of NETs-releasing cells following stimulation with plasma from patients with stage III/IV GC was higher compared with those stimulated with plasma from heathy controls and patients with stage I/II GC (P<0.001; Fig. 2B).

NETs deposited in human GC tissue. Surgical resection specimens of patients undergoing D2 radical gastrectomy for $\mathrm{GC}(\mathrm{n}=10)$ were collected. The pathological type of the GC samples was adenocarcinoma. Confocal microscopy revealed that NE and cit-h3 co-localized inside the web-structure of NETs of GC samples, with almost no expression in the control group (Fig. 3A). Western blot analysis further confirmed that the expression of cit-h3, an important biomarker of NETs, was significantly higher in GC tissues compared with normal resection margins $(\mathrm{P}<0.001$; Fig. $3 \mathrm{~B}$ and $\mathrm{C})$. The abundant deposition of NETs in tumor tissues was found to be consistent with the above-mentioned immunofluorescence results.

NETs promote the migration of GC cells. To investigate the effects of NETs on GC cells, PMA-stimulated neutrophils were used to mimic NETs formation. NETs formation was identified by evaluating the co-localization of extracellular DNA and NE by immunofluorescence microscopy (Fig. 4A). The effects of NETs on cell proliferation were assessed by measuring the viability of AGS cells by MTT assay. It was demonstrated that the cell proliferation rate did not differ significantly among the 3 groups ( $P>0.05$; Fig. 4B). Subsequently, flow cytometric analysis revealed a similar percentage of cell cycle arrest at the G2/M-phase in NETs-treated AGS cells compared with the control and NETs-suppressed groups ( $\mathrm{P}>0.05$; Fig. $4 \mathrm{C}$ and $\mathrm{D}$ ). These results indicate that NETs did not exert any effects on cell proliferation or cell cycle in the models investigated. Furthermore, the effects of NETs on the migration of AGS cells were evaluated by Transwell assay. It was demonstrated that the culture media (CM) of PMA-stimulated neutrophils significantly improved the migratory ability of AGS cells, and the pro-migratory effect was impaired by degrading/inhibiting NETs $(\mathrm{P}<0.001$; Fig. 4E and F).

NETs promote EMT in GC cells. EMT is a critical process for aggressive metastatic dissemination of cancer, which undergoes multiple and dynamic transitional states from epithelial to mesenchymal phenotypes. The present study investigated whether EMT plays a role in mediating the pro-metastatic properties of NETs by measuring the changes in the expression of theses biomarkers by western blot analysis. Following treatment with NETs CM for $24 \mathrm{~h}$, the AGS cells exhibited a decreased expression of the epithelial marker, E-cadherin, and an enhanced expression of the mesenchymal marker, vimentin. Notably, the EMT-promoting effect of the NETs were eliminated by DNAse-1/PAD4 inhibitor ( $\mathrm{P}<0.01$; Fig. 4G-I)

Effects of NETs inhibitors on tumor progression and EMT in vivo. A post-operative recurrent tumor model was used to evaluate the antitumor activity of DNAse-1/GSK-484 (Fig. 5A). The body weight of the nude mice remained stable following NETs inhibition therapy $(\mathrm{P}>0.05$; Fig. 5B). By comparing the volume and weight of tumors in each group, neither DNAse-1 nor PAD4 inhibitor significantly inhibited the growth of residual tumors ( $\mathrm{P}>0.05$; Fig. $5 \mathrm{C}$ and $\mathrm{D})$. A significant deposition of NETs was observed in the tumor sections of the NC group, as extracellular colocalization of NE with citrullinated H3; however, by contrast, a lack of deposition was observed in the DNAse-1/PAD 4 inhibitor group (Fig. 5E). To verify this observation, the expression of cit-h3 in tumor tissues was compared in each group and the results were consistent with the results of tissue fluorescence analysis ( $\mathrm{P}<0.01$; Fig. 5G and $\mathrm{J})$. In immunohistochemistry and western blot assays, the epithelial marker, E-cadherin, was found to be upregulated, while the mesenchymal marker, vimentin, was downregulated by DNAse-1/GSK-484 $(\mathrm{P}<0.01$; Fig. 5F-J). 


\section{Discussion}

In response to various stimuli, such as infections and inflammatory cytokines, neutrophils release cloudy-like structures that consist of their DNA-histone complexes decorated with antimicrobial peptides, which are termed NETs (35-37). NETs have been demonstrated to increase hypercoagulability by promoting thrombin and fibrin generation (38). However, the detailed mechanisms through which NETs cause tumor cell progression and metastasis remains unclear. The present study investigated whether NETs can promote the ability of tumor cells to migrate and if their effect can be specifically blocked by NETs inhibitors. It was identified that the tumor-promoting effect of NETs was closely associated with EMT, a key process in GC metastasis (Fig. 6).

Circulating NETs markers are significantly elevated in various types of cancer and are associated with a poor prognosis of patients $(39,40)$. It has previously been demonstrated that NETs can lead to the formation of hypercoagulable tumor states; however, the association between NETs and tumor metastasis remains unclear (41). Circulating MPO-DNA complex, cell-free DNA and NE have been proposed as NETs markers (42). The NETs levels in the peripheral blood of patients with different TNM stages GC were detected and analyzed retrospectively. It was identified that the formation capacity of NETs in GC was closely associated with the progression of the disease. It is understood that patients with advanced tumors have a higher risk of metastasis compared with patients with early-stage disease. The present study found that the level of NETs in patients with stage III/IV GC was significantly higher compared with that in healthy controls and patients with stage I/II GC, while no significant difference was found between patients with stage III and IV GC. Metastasis is more likely to occur in patients with stage III/IV GC, suggesting that the level of NETs may be associated with metastasis. Stage IV GC is notorious for distant metastasis in patients and high mortality rates, whereas no metastasis is present in patients with stage III disease. As regards the hypothesis of whether NETs play a role in tumor metastasis, the present study revealed a notable phenomenon. LVI is considered to be an independent prognostic factor for patients with GC (43). Consistent with the finding of no significant differences observed in the NETs levels in patients with stage III and IV GC, there was also no significant difference in the incidence of LVI in patients with stage III and IV GC; however, it was significantly higher compared with the other groups. These results suggest that NETs may play an important role in tumor metastasis. However, certain limitations of the present study should be acknowledged: The clinical sample size was small and insufficient to evaluate survival and determine the diagnostic significance of NETs. However, due to the limited literature in this area, it was considered important to report these results in order to encourage further studies to evaluate the effects of NETs on tumor progression.

Previous studies have demonstrated that the production of NETs in tumor patients is significantly higher compared with that in healthy controls $(44,45)$. Microenvironmental changes in tumor patients are more likely to stimulate the production of NETs, and tumor cells can release inflammatory factors, such as IL-6, IL-8 and G-CSF, to promote the release of NETs from neutrophils $(46,47)$. The present study revealed that the total number of neutrophils and the percentage of neutrophils in patients with GC did not differ significantly compared with the healthy controls; however, the level of NETs differed between the 2 groups. The TME plays an important role in tumor progression (48). We speculated that the release of NETs may be associated with the TME. Therefore, first, neutrophils were stimulated with normal plasma in the current study; however, no NETs formation was detected. Subsequently, neutrophils were stimulated with plasma from patients with different TNM stages of GC, and it was identified that plasma from patients with stage III and IV GC significantly induced the production of NETs. This was consistent with the analysis of peripheral blood. The results suggested that the level of NETs in tumor patients is associated with TME, and that NETs are an important part of the TME. Therefore, NETs may be considered as prognostic markers in patients.

Neutrophils and NETs are highly infiltrated in tumors (49). In a study on breast cancer, a high expression of NETs was identified in high metastatic triple-negative breast cancer tissues, with a lower expression in HER-2 type breast cancer, and almost no NETs were observed in luminal breast cancer. It was also revealed that this differential expression of NETs was positively associated with the degree of metastasis and closely associated with the prognosis of patients (13). The present study first reported the high expression of NETs in GC tissues. Subsequently, a comparison was made between $\mathrm{GC}$ tissues and the normal resection margins. It was identified that the expression of NETs in tumor tissues was significantly increased, while NETs could hardly be detected in normal tissues. A large extent of NETs deposition in tumors may serve as a promoter of tumor progression and metastasis. NETs, whose main components are cell-free DNA and histones, have been reported to induce tumor metastasis by capturing circulating free tumor cells (15). In addition to promoting metastasis, NETs are also considered to play an antitumor role in certain tumors, and have been reported to inhibit the proliferation and promote the apoptosis of colon cancer cells (19). The antitumor effect of NETs was also reported in a study on melanoma (50). It was hypothesized that NETs play a dual role in tumor progression. On the one hand, NETs can inhibit tumor progression as a continuation of initial inflammatory response. On the other hand, NETs may play an adverse role in tumor progression offering a scaffold with many molecules with biological activity, which may promote malignant cells progression and migration (15). When the melanoma cell line A375 was stimulated with NETs, their ability to metastasize and proliferate declined. However, this antitumor effect can be reversed by DNase I (20). Moreover, the web-like structure promotes the adhesion of melanoma cells similar to the mechanism for capturing microbes (36). In addition, NETs were found to inhibit cancer cell growth by inducing apoptosis of Caco-2 and AML cells (19). However, current studies on the antitumor effects of NETs lack in-depth studies on the mechanisms, which are presented in terms of phenotypes. Therefore, the present study investigated the effects of NETs on GC cells. By stimulating tumor cells with NETs-containing culture medium or a NETs inhibitor to observe the effects of NETs on cell migration, it was identified that the specific tumor-promoting effect of NETs were blocked by NET inhibitor. DNase-1 is a 
powerful inhibitor that degrades NETs by degrading the DNA structure. PAD4 is a key enzyme involved in the release of NETs into the extracellular domain, and inhibition of PAD4 has been reported to inhibit chromatin depolymerization and thereby inhibit the the release of NETs into the extracellular domain. The present study confirmed through in vitro experiments that both inhibitors can effectively inhibit the formation of NETs and the migration of tumor cells, to play a specific antitumor role. However, the limitations of NETs on other aspects of tumor cells need to be further verified. The current results suggested that NETs inhibitors may have some practical application value in the treatment and prevention of tumor metastasis.

EMT is a prerequisite and important process for the metastasis of GC (51). Following the loss of the epithelial phenotype and the gain of mesenchymal features, tumor cells acquire a potent ability to migrate and invade which then leads to distant metastasis. Neutrophils have been reported to induce EMT in tumor cells. When tumor cells were stimulated by neutrophils, the expression of classical EMT markers, such as slug and vimentin, were upregulated (52). Hu et al (53) reported that the number of infiltrated neutrophils in tumor cells was negatively associated with the expression of the epithelial marker E-cadherin in lung cancer tissues. The present study found a large number of NETs in patients with advanced GC with high risk of metastasis. Stimulation of GC cells with NETs-containing medium inhibited the expression of epithelial marker, E-cadherin, enhanced the expression of the stromal marker, vimentin, and promoted the migration of GC cells. This pro-EMT effect was attenuated by the NETs inhibitors, and this specific effect may provide novel strategies for the treatment of tumor metastasis. The occurrence of neutrophil-induced EMT is associated with numerous signaling pathways, such as the AKT signaling pathway, the TGF- $\beta$ /Smad signaling pathway and the ERK signaling pathway (53-55). Whether NETs can be activated via relevant signaling pathways to promote EMT in GC cells remains to be further verified.

In addition, the NETs promoted cell migration through EMT is probability mediated by NETs-associated proteases. The NETs DNA bound to the extracellular matrix (ECM), thus bringing NETs associated proteases to their substrate (27). The NETs-mediated proteolytic remodeling of ECM may cause many epitope exposures that affected proliferation or cell cycle change of cancer cells through various signaling pathway, such as integrin and Ras/Raf/MAPK signaling (27,56). In addition, STAT1/STAT3/STAT5 and cyclin-dependent-kinase (CDKs) are both involved in the regulation of cell cycle and cell proliferation $(57,58)$. Whether NETs induce cell proliferation and cell cycle changes in these ways remains to be tested. It may be a potent strategy to prevent cancer recurrence and broadly serve as treatment for other NETs associated pathological processes, such as EMT. If such an association exists, it may be possible that NETs and their downstream effectors could be targeted to reduce the risk of tumor metastasis. The mechanisms of the effects of NETs on tumor progression will be the focus of our future research.

In conclusion, the present study revealed a novel pro-tumor activity of neutrophils mediated by NETs, which may explain the poor prognosis of patients with GC associated with neutrophil aggregation within the tumor environment. As presented in Fig. 6, GC primes neutrophils towards NETosis, then NETs deposit in GC tissues or adhere to epithelial cancer cells, notably without affecting cell proliferation and cell cycle, and NETs promote cancer cell migration via EMT in vitro and in vivo.

The number of NETs in peripheral blood was increased significantly in patients with advanced GC and was positively associated with the increased risk of tumor staging and metastasis. NETs are expected to become a novel tumor biomarker and provide a novel therapeutic target for the comprehensive treatment of GC. In the future, NETs inhibitors may become a promising drug which may be used to prevent tumor recurrence.

\section{Acknowledgements}

The authors would like to thank Professor Chu Wenfeng of Harbin Medical University for providing guidance on the experimental techniques and for the provision of the experimental instruments.

\section{Funding}

The present study was supported by the National Science Foundation of China (grant no. 81672355).

\section{Availability of data and materials}

The datasets generated and analyzed during the present study are available from the corresponding author on reasonable request.

\section{Authors' contributions}

TZ designed the study, performed some experiments, analyzed the data, prepared the figures and wrote the manuscript. XZ obtained funding, designed the study, performed some experiments and revised the manuscript. LL, BW, HL and ZX conducted the mouse experiments, cell culture and cell treatments. CY, $\mathrm{RL}, \mathrm{DH}$ and QW performed some experiments and analyzed some of the results. All authors were substantially involved in the research, acquisition of the data, analysis and manuscript preparation. All authors read and approved the final manuscript.

\section{Ethics approval and consent to participate}

All procedures performed in studies involving human participants were in accordance with the standards upheld by the Ethics Committee of the Second Affiliated Hospital of Harbin Medical University and with those of the 1964 Helsinki Declaration and its later amendments for ethical research involving human subjects. All subjects manually signed the informed consent. All animal experiments were performed in accordance with the NIH guidelines (Guide for the Care and Use of Laboratory Animals) and approved by the Ethics Committee of the Second Affiliated Hospital of Harbin Medical University, P.R. China. The Ethics Committee of the Second Affiliated Hospital of Harbin Medical University approved the present study (permit no. KY2016-032). 


\section{Patient consent for publication}

Not applicable.

\section{Competing interests}

The authors declare that they have no competing interests.

\section{References}

1. Bray F, Ferlay J, Soerjomataram I, Siegel RL, Torre LA and Jemal A: Global cancer statistics 2018: GLOBOCAN estimates of incidence and mortality worldwide for 36 cancers in 185 countries. CA Cancer J Clin 68: 394-424, 2018.

2. Chen W, Zheng R, Baade PD, Zhang S, Zeng H, Bray F, Jemal A Yu XQ and He J: Cancer statistics in China, 2015. CA Cancer J Clin 66: 115-132, 2016.

3. Arneth B: Tumor microenvironment. Medicina (Kaunas) 56: 15, 2019.

4. Quail DF and Joyce JA: Microenvironmental regulation of tumor progression and metastasis. Nat Med 19: 1423-1437, 2013.

5. Gonzalez H, Hagerling $\mathrm{C}$ and Werb Z: Roles of the immune system in cancer: From tumor initiation to metastatic progression. Genes Dev 32: 1267-1284, 2018.

6. Zhang $\mathrm{X}$, Shi $\mathrm{H}$, Yuan X, Jiang $\mathrm{P}$, Qian $\mathrm{H}$ and $\mathrm{Xu} \mathrm{W}$ : Tumor-derived exosomes induce N2 polarization of neutrophils to promote gastric cancer cell migration. Mol Cancer 17: 146, 2018.

7. Shaul ME and Fridlender ZG: Tumour-associated neutrophils in patients with cancer. Nat Rev Clin Oncol 16: 601-620, 2019.

8. Coffelt SB, Wellenstein MD and de Visser KE: Neutrophils in cancer: Neutral no more. Nat Rev Cancer 16: 431446, 2016.

9. Liu Y and Liu L: The pro-tumor effect and the anti-tumor effect of neutrophils extracellular traps. Biosci Trends 13: 469-475, 2020.

10. Demers M, Krause DS, Schatzberg D, Martinod K, Voorhees JR, Fuchs TA, Scadden DT and Wagner DD: Cancers predispose neutrophils to release extracellular DNA traps that contribute to cancer-associated thrombosis. Proc Natl Acad Sci USA 109 13076-13081, 2012

11. Cedervall J, Hamidi A and Olsson AK: Platelets, NETs and cancer. Thromb Res 164 (Suppl 1): S148-S152, 2018.

12. Berger-Achituv S, Brinkmann V, Abed UA, Kühn LI, Ben-Ezra J, Elhasid R and Zychlinsky A: A proposed role for neutrophil extracellular traps in cancer immunoediting. Front Immunol 4 48, 2013.

13. Park J, Wysocki RW, Amoozgar Z, Maiorino L, Fein MR, Jorns J, Schott AF, Kinugasa-Katayama Y, Lee Y, Won NH, et al: Cancer cells induce metastasis-supporting neutrophil extracellular DNA traps. Sci Transl Med 8: 361ra138, 2016.

14. Najmeh S, Cools-Lartigue J, Rayes RF, Gowing S, Vourtzoumis $P$, Bourdeau F, Giannias B, Berube J, Rousseau S, Ferri LE and Spicer JD: Neutrophil extracellular traps sequester circulating tumor cells via $\beta 1$-integrin mediated interactions. Int J Cancer 140: 2321-2330, 2017.

15. Cools-Lartigue J, Spicer J, McDonald B, Gowing S, Chow S, Giannias B, Bourdeau F, Kubes P and Ferri L: Neutrophil extracellular traps sequester circulating tumor cells and promote metastasis. J Clin Invest 123: 3446-3458, 2013.

16. Grilz E, Mauracher LM, Posch F, Königsbrügge O, Zöchbauer-Müller S, Marosi C, Lang I, Pabinger I and Ay C: Citrullinated histone $\mathrm{H} 3$, a biomarker for neutrophil extracellular trap formation, predicts the risk of mortality in patients with cancer. Br J Haematol 186: 311-320, 2019.

17. Erpenbeck L and Schön MP: Neutrophil extracellular traps: Protagonists of cancer progression? Oncogene 36: 2483-2490, 2017.

18. Claushuis TA, de Stoppelaar SF, Stroo I, Roelofs JJ, Ottenhoff R, van der Poll $\mathrm{T}$ and Van't Veer C: Thrombin contributes to protective immunity in pneumonia-derived sepsis via fibrin polymerization and platelet-neutrophil interactions. J Thromb Haemost 15: 744-757, 2017.

19. Arelaki S, Arampatzioglou A, Kambas K, Papagoras C, Miltiades P, Angelidou I, Mitsios A, Kotsianidis I, Skendros P, Sivridis E, et al: Gradient infiltration of neutrophil extracellular traps in colon cancer and evidence for their involvement in tumour growth. PLoS One 11: e0154484, 2016.
20. Schedel F, Mayer-Hain S, Pappelbaum KI, Metze D, Stock M, Goerge T, Loser K, Sunderkötter C, Luger TA and Weishaupt C: Evidence and impact of neutrophil extracellular traps in malignant melanoma. Pigment Cell Melanoma Res 33: 63-73, 2020.

21. Gupta GP and Massagué J: Cancer metastasis: Building a framework. Cell 127: 679-695, 2006.

22. Pastushenko I and Blanpain C: EMT transition states during tumor progression and metastasis. Trends Cell Biol 29: 212-226, 2019.

23. Zhang W, Gu J, Chen J, Zhang P, Ji R, Qian H, Xu W and Zhang $\mathrm{X}$ : Interaction with neutrophils promotes gastric cancer cell migration and invasion by inducing epithelial-mesenchymal transition. Oncol Rep 38: 2959-2966, 2017.

24. Li S, Cong X, Gao H, Lan X, Li Z, Wang W, Song S, Wang Y, Li C, Zhang H, et al: Correction to: Tumor-associated neutrophils induce EMT by IL-17a to promote migration and invasion in gastric cancer cells. J Exp Clin Cancer Res 38: 177, 2019.

25. Rayes RF, Vourtzoumis P, Bou Rjeily M, Seth R, Bourdeau F, Giannias B, Berube J, Huang YH, Rousseau S, Camilleri-Broet S, et al: Neutrophil extracellular trap-associated CEACAM1 as a putative therapeutic target to prevent metastatic progression of colon carcinoma. J Immunol 204: 2285-2294, 2020.

26. American Joint Committee on Cancer (AJCC): Cancer Staging Manual. AJCC, Chicago, IL, 2016. https://cancerstaging.org/ references-tools/deskreferences/pages/default.aspx.

27. Albrengues J, Shields MA, Ng D, Park CG, Ambrico A, Poindexter ME, Upadhyay P, Uyeminami DL, Pommier A, Küttner V, et al: Neutrophil extracellular traps produced during inflammation awaken dormant cancer cells in mice. Science 361: eaao4227, 2018.

28. Li B, Liu Y, Hu T, Zhang Y, Zhang C, Li T, Wang C, Dong Z, Novakovic VA, $\mathrm{Hu} \mathrm{T}$ and Shi J: Neutrophil extracellular traps enhance procoagulant activity in patients with oral squamous cell carcinoma. J Cancer Res Clin Oncol 145: 1695-1707, 2019.

29. Hu Y, Li H, Yan R, Wang C, Wang Y, Zhang C, Liu M, Zhou T, Zhu W, Zhang H, et al: Increased neutrophil activation and plasma DNA levels in patients with pre-eclampsia. Thromb Haemost 118: 2064-2073, 2018.

30. Schimmel M, Nur E, Biemond BJ, van Mierlo GJ, Solati S, Brandjes DP, Otten HM, Schnog JJ and Zeerleder S; Curama Study Group: Nucleosomes and neutrophil activation in sickle cell disease painful crisis. Haematologica 98: 1797-1803, 2013.

31. Yoo DG, Floyd M, Winn M, Moskowitz SM and Rada B: NET formation induced by Pseudomonas aeruginosa cystic fibrosis isolates measured as release of myeloperoxidase-DNA and neutrophil elastase-DNA complexes. Immunol Lett 160: 186-194, 2014.

32. Stakos DA, Kambas K, Konstantinidis T, Mitroulis I, Apostolidou E, Arelaki S, Tsironidou V, Giatromanolaki A, Skendros P, Konstantinides S and Ritis K: Expression of functional tissue factor by neutrophil extracellular traps in culprit artery of acute myocardial infarction. Eur Heart J 36: 1405-1414, 2015.

33. Abed AU and Brinkmann V: Immunofluorescence labelling of human and murine neutrophil extracellular traps in paraffin-embedded tissue. J Vis Exp 151: e60115, 2019.

34. Owen GR, Häkkinen L, Wu C and Larjava $\mathrm{H}$ : A reproducible technique for specific labeling of antigens using preformed fluorescent molecular IgG-F(ab') 2 complexes from primary antibodies of the same species. Microsc Res Tech 73: 623-630, 2010.

35. Hu Z, Murakami T, Tamura H, Reich J, Kuwahara-Arai K, Iba T, Tabe Y and Nagaoka I: Neutrophil extracellular traps induce IL-1 $\beta$ production by macrophages in combination with lipopolysaccharide. Int J Mol Med 39: 549-558, 2017.

36. Brinkmann V, Reichard U, Goosmann C, Fauler B, Uhlemann Y, Weiss DS, Weinrauch Y and Zychlinsky A: Neutrophil extracellular traps kill bacteria. Science 303: 1532-1535, 2004.

37. Nauseef WM and Borregaard N: Neutrophils at work. Nat Immunol 15: 602-611, 2014

38. Yang C, Sun W, Cui W, Li X, Yao J, Jia X, Li C, Wu H, Hu Z and Zou X: Procoagulant role of neutrophil extracellular traps in patients with gastric cancer. Int J Clin Exp Pathol 8: 14075-14086, 2015.

39. Marin Oyarzún CP, Carestia A, Lev PR, Glembotsky AC Castro Ríos MA, Moiraghi B, Molinas FC, Marta RF, Schattner M and Heller PG: Neutrophil extracellular trap formation and circulating nucleosomes in patients with chronic myeloproliferative neoplasms. Sci Rep 6: 38738, 2016. 
40. Oklu R, Sheth RA, Wong KHK, Jahromi AH and Albadawi H: Neutrophil extracellular traps are increased in cancer patients but does not associate with venous thrombosis. Cardiovasc Diagn Ther 7(Suppl 3): S140-S149, 2017.

41. Guglietta S and Rescigno M: Hypercoagulation and complement: Connected players in tumor development and metastases. Semin Immunol 28: 578-586, 2016.

42. Brinkmann V: Neutrophil extracellular traps in the second decade. J Innate Immun 10: 414-421, 2018.

43. Wu L, Liang Y, Zhang C, Wang X, Ding X, Huang C and Liang H: Prognostic significance of lymphovascular infiltration in overall survival of gastric cancer patients after surgery with curative intent. Chin J Cancer Res 31: 785-796, 2019.

44. Abdol Razak N, Elaskalani O and Metharom P: Pancreatic cancer-induced neutrophil extracellular traps: A potential contributor to cancer-associated thrombosis. Int J Mol Sci 18 487, 2017.

45. Richardson JJR, Hendrickse C, Gao-Smith F and Thickett DR Neutrophil extracellular trap production in patients with colorectal cancer in vitro. Int J Inflam 2017: 4915062, 2017.

46. Zhang Y, Wang C, Yu M, Zhao X, Du J, Li Y, Jing H, Dong Z, Kou J, Bi Y, et al: Neutrophil extracellular traps induced by activated platelets contribute to procoagulant activity in patients with colorectal cancer. Thromb Res 180: 87-97, 2019.

47. Arpinati L, Shaul ME, Kaisar-Iluz N, Mali S, Mahroum S and Fridlender ZG: NETosis in cancer: A critical analysis of the impact of cancer on neutrophil extracellular trap (NET) release in lung cancer patients vs. mice. Cancer Immunol Immunother 69: 199-213, 2020

48. Wu L, Saxena S and Singh RK: Neutrophils in the tumor microenvironment. Adv Exp Med Biol 1224: 1-20, 2020.

49. Hisada Y, Grover SP, Maqsood A, Houston R, Ay C, Noubouossie DF, Cooley BC, Wallén $\mathrm{H}$, Key NS, Thålin C, et al: Neutrophils and neutrophil extracellular traps enhance venous thrombosis in mice bearing human pancreatic tumors. Haematologica 105: 218-225, 2020.
50. Andzinski L, Kasnitz N, Stahnke S, Wu CF, Gereke M, von Köckritz-Blickwede M, Schilling B, Brandau S, Weiss S and Jablonska J: Type I IFNs induce anti-tumor polarization of tumor associated neutrophils in mice and human. Int $\mathrm{J}$ Cancer 138: 1982-1993, 2016.

51. Natalwala A, Spychal R and Tselepis C: Epithelial-mesenchymal transition mediated tumourigenesis in the gastrointestinal tract. World J Gastroenterol 14: 3792-3797, 2008.

52. Wang Y, Chen J, Yang L, Li J, Wu W, Huang M, Lin L and Su S: Tumor-contacted neutrophils promote metastasis by a CD90-TIMP-1 Juxtacrine-paracrine loop. Clin Cancer Res 25: 1957-1969, 2019.

53. Hu P, Shen M, Zhang $P$, Zheng $C$, Pang Z, Zhu L and $\mathrm{Du}$ J: Intratumoral neutrophil granulocytes contribute to epithelial-mesenchymal transition in lung adenocarcinoma cells. Tumour Biol 36: 7789-7796, 2015.

54. Wu Y, Zhao Q, Peng C, Sun L, Li XF and Kuang DM: Neutrophils promote motility of cancer cells via a hyaluronan-mediatedTLR4/PI3K activation loop. J Pathol 225: 438-447, 2011.

55. Zhou Q, Wang X, Yu Z, Wu X, Chen X, Li J, Zhu Z, Liu B and $\mathrm{Su}$ L: Transducin $(\beta)$-like $1 \mathrm{X}$-linked receptor 1 promotes gastric cancer progression via the ERK1/2 pathway. Oncogene 36: 1873-1886, 2017.

56. Molina JR and Adjei AA: The Ras/Raf/MAPK pathway. J Thorac Oncol 1: 7-9, 2006.

57. Groner B and von Manstein V: Jak Stat signaling and cancer: Opportunities, benefits and side effects of targeted inhibition. Mol Cell Endocrinol 451: 1-14, 2017.

58. Lim S and Kaldis P: Cdks, cyclins and CKIs: Roles beyond cell cycle regulation. Development 140: 3079-3093, 2013. International (CC BY-NC-ND 4.0) License. 